\title{
Bariatric Surgery Impact on Sickle Cell Disease Pain Crisis: A Case Report
}

\author{
Hassan Al-Jafar ${ }^{1}$, Masouma Al-Ali², Osama R. Kombar ${ }^{3}$, Mohammad Alhaifi ${ }^{4}$ \\ ${ }^{1}$ Hematology Department, Amiri Hospital, Kuwait City, Kuwait \\ ${ }^{2}$ Liver \& Gastroenterology Department, Amiri Hospital, Kuwait City, Kuwait \\ ${ }^{3}$ Radiologist Department, Amiri Hospital, Kuwait City, Kuwait \\ ${ }^{4}$ Surgery Department, Amiri Hospital, Kuwait City, Kuwait \\ Email: `haj400004@gmail.com,dr.masouma1@gmail.com, ukombar@yahoo.com,dralhaifi@hotmail.com
}

How to cite this paper: Al-Jafar, $\mathrm{H}$., Al-Ali, M., Kombar, O.R. and Alhaifi, M. (2020) Bariatric Surgery Impact on Sickle Cell Disease Pain Crisis: A Case Report. Case Reports in Clinical Medicine, 9, 158-164. https://doi.org/10.4236/crcm.2020.96023

Received: May 20, 2020

Accepted: June 14, 2020

Published: June 17, 2020

Copyright ( 2020 by author(s) and Scientific Research Publishing Inc. This work is licensed under the Creative Commons Attribution International License (CC BY 4.0).

http://creativecommons.org/licenses/by/4.0/

(c) (i) Open Access

\begin{abstract}
Sickle cell disease (SCD) is an autosomal genetic blood disorder resulting in multiple end-organ complications. Malnutrition is a stress factor that can cause a SCD crisis. Bariatric surgery is a weight reduction surgery that involves the binding or removing a part of the stomach or resecting and re-routing the small intestine to a small stomach pouch. It is known to cause malnutrition and stress. Malnutrition affects more than two billion people of all ages worldwide due to different causes. Long-term deficiency of micronutrients leads to reduced immunity, leukopenia, and diseases affecting the psychological, skeletal, and central nervous system. We here present the case of a 20-year-old woman with SCD and class III obesity. She underwent sleeve gastrectomy in 2018 following psychological distress caused by being severely overweight. She had mild SCD pain, but after the bariatric surgery, it became severe, requiring morphine treatment and monthly exchange transfusion beside the NSAID which become not much effective as before bariatric surgery. Our findings show that bariatric surgery, which leads to a stressful condition, can aggravate the SCD pain crisis, thereby highlighting the need for alternative methods of weight reduction in these patients. Controlled studies are required for the proper assessment of bariatric surgery in SCD.
\end{abstract}

\section{Keywords}

Sickle Cell Disease, Bariatric Surgery, Pain Crisis

\section{Introduction}

Sickle cell disease (SCD) is an autosomal genetic blood disorder leading to severe anemia and impaired tissue perfusion, resulting in multiple end-organ compli- 
cations. Pain is the most common complication of SCD. Some SCD patients have mild to moderate pain, while others have severe vaso-occlusive complications, which lead to a pain crisis. Poor hydration increases blood viscosity. Even mild dehydration can have a significant impact on hemoglobin concentration [1]. Stress factors that affect the SCD pain crisis are fever, loss of fluids due to excessive sweating, vomiting, diarrhea, malnutrition, high temperature, and humidity. The renal complications in SCD can impact the urine concentration resulting in higher urine loss and dehydration due to tubular damage [2].

Bariatric surgery (BS) is a weight reduction surgery that involves binding or removing part of the stomach or resecting and re-routing the small intestine to a stomach pouch. The United States National Institutes of Health recommends BS for individuals with a body mass index (BMI) $\geq 40$ and those with a BMI of $\geq 35$ with comorbidities such as diabetes and other obesity-related severe disorders [3]. All forms of BS reduce food intake and the quality of micronutrients absorption. Following BS, appropriate incorporation of vitamins and minerals can help maintain normal metabolic functions, energy production, hormone synthesis, and decrease the risk of nutrient-related health problems [4]. In bodyweight calculations, a BMI of <18.5 falls within the underweight range, between 18.5 and $<25$ is the normal range, between 25.0 and $<30$ is the overweight range, and $<30.0$ falls within the obese range. Obesity is frequently subdivided into categories: Class 1: BMI of 30 to <35, Class 2: BMI of 35 to $<40$, Class 3: BMI of 40 or higher. Class 3 obesity is sometimes categorized as "extreme" or "severe" obesity [5].

Micronutrient deficiencies cause several diseases while exacerbating many others and affect more than two billion people of all ages. It is associated with $10 \%$ of all deaths in children and child welfare. Lack of one or several micronutrients is caused by its inadequate intake, malabsorption, increased requirements, and drug-nutrient interactions. In about $70 \%$ of the children and $40 \%$ of the adults, these deficiencies cause complications [6]. In many cases, the response to a nutrient deficiency also seems to be genotype-specific. Gene-nutrient interactions are thus a fascinating example of physiological reactions to the environment and diet at the molecular level [7]. Long-term deficiency of micronutrients could cause many disorders, including reduced immunity and leukopenia. Gastrointestinal, central nervous system, psychological, skeletal, and reproductive systems are most affected by these deficiencies [8]. This study aimed to highlight that bariatric surgery is not suitable for all morbidity obese people, and more diseases should be added to the contraindications list of BS.

\section{Case Study}

The patient was a 20-year-old woman with SCD. She weighed 104 kilograms, was $156 \mathrm{~cm}$ tall, and had a BMI of 42 . She had neither hypertension nor any metabolic and other chronic disorders. The indication for this surgery was the severe psychological distress from being grade III overweight, and the failure to 
reduce weight with many other measures. Although she had SCD, she was never under follow up with a hematologist as she only had mild chronic SCD pain, which was controlled with nonsteroidal anti-inflammatory drugs (NSAIDs). She underwent lap sleeve gastrectomy in 2018, after she signed a consent form. On two weeks post the BS, she was admitted with vomiting and abdominal pain; subsequently, she required several admissions because of dehydration and severe body pain. The SCD pain crisis became severe, requiring morphine to control the pain. She was given intravenous fluids on several occasions. Lately, she has been on a monthly exchange transfusion to control the SCD pain and to reduce the morphine requirement. Her lab findings before and after the BS are as follows:

Before the BS, complete blood count (CBC) on 29-08-2017: White blood cells (WBCs): $17.5(4-10) \times 10^{9} / \mathrm{L}$, red blood cells $(\mathrm{RBC}): 3.89(3.8-4.8) \times 10^{12} / \mathrm{L}$, hemoglobin: $111(120-150) \mathrm{g} / \mathrm{L}$, hematocrit: $0.384(0.36-0.46) \mathrm{L} / \mathrm{L}$, platelet count: $523(150-410) \times 10 \%$, MCV: $90(83-101)$ fl, MCH: $29(27-32) \mathrm{pg}$, MCHC: 348 (315 - 345) g/L, and reticulocytes: 11.6 (0.5 - 2.5)\%. Coagulation profile: INR: 1.35 and APTT: 35.2 (27 - 38.3) sec. Serum ferritin: 95.1 (11 - 306) $\mathrm{ng} / \mathrm{L} . \mathrm{Hb}$ electrophoresis: $\mathrm{Hb} \mathrm{A}=0 \%, \mathrm{Hb} \mathrm{F}=26.1 \% \mathrm{Hb} \mathrm{A} 2=1.3 \%$, and $\mathrm{Hb} \mathrm{S}=$ 72.6\%. G6PD: normal. Liver function test: Total protein: $73(61-79) \mathrm{g} / \mathrm{L}$, albumin: $40(35$ - 48) g/L, total bilirubin: $82(3-25)$ Umol/L, direct biluribin: 26.2 (1.7 - 8.6) Umol/L, LDH: 528 IU/L, ALT: 79 (10 - 60) IU/L, AST: 85 (10 - 42) IU/L, glucose: $5.0(3.9-6.1) \mathrm{mmol} / \mathrm{L}$, and Ca: 2.17 (2.1 - 2.6) mmol/L. Renal function test: BUN: $0.9(2.5$ - 7.1) mmol/L, creatinine: $56(26-77)$ umol/L, Na: $138(134$ - 144) mmol/L, K: 4.03 (3.6 - 5.1) mmol/L, alkaline phoshatase: 142 (42 - 98) IU/L, corrected Ca: $2.17 \mathrm{mmol} / \mathrm{L}$.

Abdominal ultrasound before the BS was not available.

Eighteen months after the BS, her body weight, height and BMI were $65 \mathrm{~kg}$, $155 \mathrm{~cm}$, and 26.7, respectively. Other lab findings were as follows:

WBC: $12.2(3.8-4.8) \times 10^{12} / \mathrm{L}, \mathrm{RBC}: 3.08 \times 10^{12} / \mathrm{L}$, hemoglobin: $93(120-150)$ g/L, hematocrit: $0.271(0.36-0.46) \mathrm{L} / \mathrm{L}$, platelet count: $322(150-410) \times 10^{9} \%$, MCV: 88 (83 - 101) fl, MCH: 30 (27 - 32) pg, MCHC: 343 (315 - 345) g/L, and reticylocytes: $6.67(0.5-2.5) \%$. Coagulation profile: INR: 1.22 and APTT: 30.7 (27 - 38.3) sec. Serum ferritin: $269.5 \mathrm{ng} / \mathrm{L}(11$ - 306) ng/L.

Liver function test: Total protein: $58(61-79) \mathrm{g} / \mathrm{L}$, albumin: $33(35-48) \mathrm{g} / \mathrm{L}$, total bilirubin: 91 (3 - 25) Umol/L, direct biluribin: 10.6 (1.7 - 8.6) Umol/L, ALT: 17 (10 - 60) IU/L, AST: 36 (10 - 42) IU/L, glucose: $4.6(3.9-6.1) \mathrm{mmol} / \mathrm{L}$, and Ca: $2.08(2.1-2.6) \mathrm{mmol} / \mathrm{L}$.

Renal function test: BUN: $<0.4(2.5$ - 7.1) mmol/L, creatinine: 48 (26 - 77) umol/L, Na: 142 (134 - 144) mmol/L, K: 3.35 (3.6 - 5.1) mmol/L, alkaline phosphatase: 67 (42 - 98) IU/L, and corrected Ca: $2.22 \mathrm{mmol} / \mathrm{L}$.

An abdominal ultrasound after the BS revealed mild hepatomegaly, mild splenomegaly, and cholecystectomy. The enlarged spleen indicated suspected old splenic infarctions due to SCD. There were no indications of intra-abdominal abscess or collections (Figure 1). 


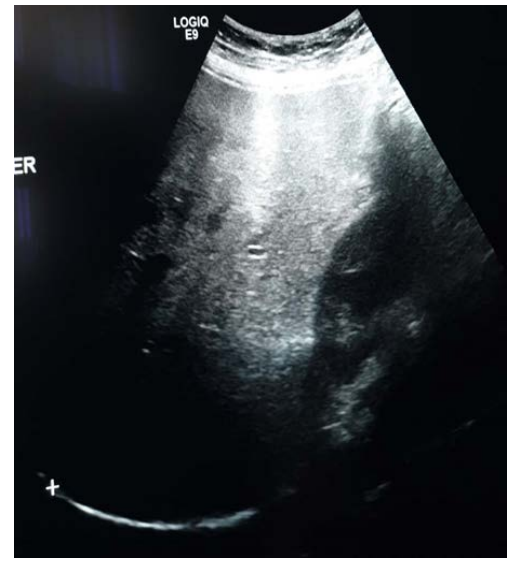

(a)

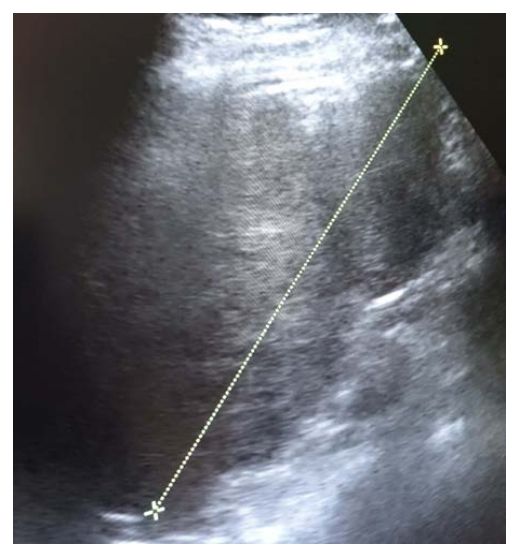

(c)

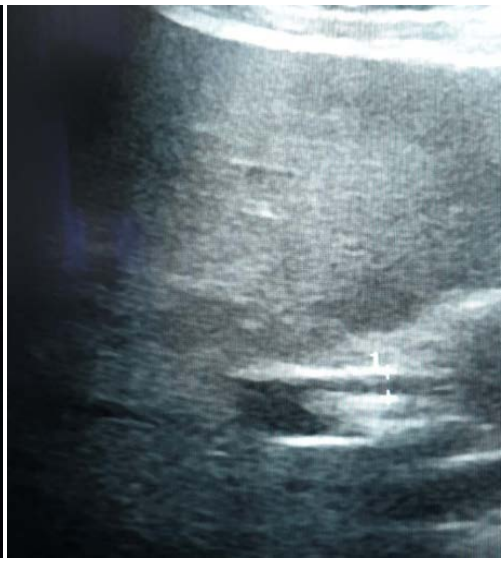

(b)

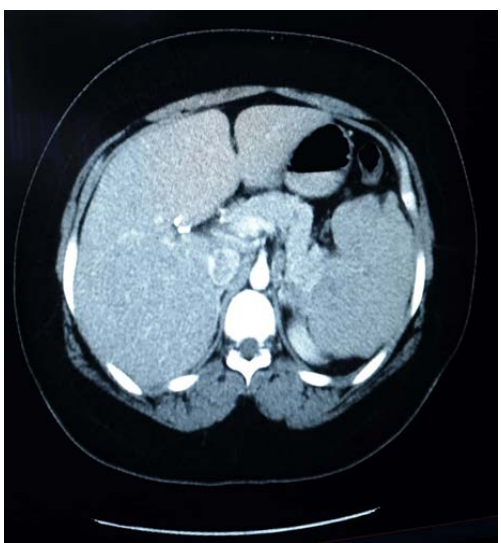

(d)

Figure 1. Abdominal ultrasound \& CT. (a) Mildly enlarged bright liver measuring about $20 \mathrm{~cm}$ in the mid-clavicular craniocaudal dimension; (b) Removed gall bladder with normal diameter of CBD measuring about $5 \mathrm{~mm}$; (c) Enlarged spleen measuring about $14 \mathrm{~cm}$ in craniocaudal dimension with irregular outline due to old infarcts; (d) Axial CT image showing hepatosplenomegaly surgical clips of cholecystectomy irregular outline of the.

The patient currently weighs $65 \mathrm{~kg}$, but she has frequent episodes of severe SCD pain, which require morphine and monthly exchange transfusion.

\section{Discussion}

A SCD patient is a fragile person who needs to stay well hydrated and away from any stress, including psychological, to avoid a painful vaso-occlusive crisis. Common complaints following BS include lethargy, easy fatigue, headaches, and muscle wasting. Reduced food intake is a stressor and contributes to SCD pain and other SCD-related complications [9]. BS deprives the body of water, which increases the blood viscosity resulting in more frequent and severe episodes of SCD crisis.

The aberrant migration of potassium and chloride ions across the RBC membrane causes RBC dehydration, which consequently increases the tendency of hemoglobin to polymerize and sickle [10]. After BS, patients usually develop 
dumping syndrome, characterized by abdominal pain, nausea, vomiting, diarrhea, and diaphoresis [11]. A few months or few years after BS, the patients become deficient in micronutrients and trace elements including water, fat-soluble elements such as iron, calcium, phosphorus, copper, magnesium, selenium, chromium, and zinc [12], as well as vitamins such as thiamine, riboflavin, niacin, pantothenic acid, pyridoxine, biotin, folate, cobalamin and vitamins $\mathrm{B}, \mathrm{D}, \mathrm{C}, \mathrm{E}$, $\mathrm{K}$. These nutrients and elements work as enzymatic cofactors in several metabolic processes and biochemical reactions. Therefore, BS can cause a wide range of nutritional, systemic, and psychiatric complications [13]. Arginine and L-glutamine, which are essential micronutrients in SCD, can get severely depleted and influence both the pain intensity and general health of the SCD patients [14]. Proteins are key regulators of metabolism and DNA replication. They also aid in digestion as well as combating infections by strengthening the immune system [15]. The specific risk for developing micronutrient and protein deficiencies cannot be predicted as there is no consensus on the amount of vitamin and mineral supplementation required after BS. However, it is clear that micronutrient deficiencies are relatively common in patients after all types of BS, and so, it is important to screen them before and periodically after the surgery for micronutrients and trace elements [12]. For any type of surgery, SCD patients need special hematological recommendations for maintaining adequate blood level, oxygen saturation, and proper hydration before and after the surgery [16]. Surgical clearance is required from a hematologist before the surgery to avoid any unexpected complications. Pre-operative assessment must include a careful review of the patient's known crisis triggers, baseline hematological profile, transfusion requirements, pre-existing organ dysfunctions, pain degree, and opioid consumption.

\section{Conclusion}

Compared to healthy individuals, those with SCD have a much lower tolerance for stress. Bariatric surgery puts these patients in a severely stressful condition. This case is significant for both areas of hematology and bariatric surgery. In this study, the SCD pain crisis worsened following the sleeve gastrectomy surgery, indicating that SCD could be a contraindication for such surgery. This case emphasized that bariatric surgery is not suitable for all obese overweight people. Controlled studies are required to assess and define the guideline to improve the outcomes of bariatric surgery, which is now a standard procedure in many countries.

\section{Acknowledgements}

This research work done under the "sickle cell disease classification of complication" project which has Ministry Of Health ethical approval number DVR/906, and funded "Partially" by Kuwait Foundation for the advancement of science under code: P116-13MM-01. 


\section{Conflicts of Interest}

The authors have no conflict of interest in this study.

\section{References}

[1] Williams-Hooker, R. (2013) Evaluation of Hydration Status, Sodium and Fluid Intake in Children with Sickle Cell Anaemia. Journal of Blood Disorders \& Transfusion, 4, 143. https://doi.org/10.4172/2155-9864.1000143

[2] Revuelta, K.L. and Andrés, M.P.R. (2011) Kidney Abnormalities in Sickle Cell Disease. Nefrologia, 31, 591-601.

[3] Robinson, M.K. (2009) Surgical Treatment of Obesity-Weighing the Facts. The New England Journal of Medicine, 361, 520-521. https://doi.org/10.1056/NEJMe0904837

[4] Ahmed, S.G. (2011) The Role of Infection in the Pathogenesis of Vaso-Occlusive Crisis in Patients with Sickle Cell Disease. Mediterranean Journal of Hematology and Infectious Diseases, 3, e2011028. https://doi.org/10.4084/mjhid.2011.028

[5] Skinner, A.C. and Skelton, J.A. (2014) Prevalence and Trends in Obesity and Severe Obesity among Children in the United States, 1999-2012. JAMA Pediatrics, 168, 561-566.

http://archpedi.jamanetwork.com/article.aspx?doi=10.1001/jamapediatrics.2014.21 https://doi.org/10.1001/jamapediatrics.2014.21

[6] Hwalla, N., Al Dhaheri, A.S., Radwan, H., et al. (2017) The Prevalence of Micronutrient Deficiencies and Inadequacies in the Middle East and Approaches to Interventions. Nutrients, 9, 229. https://doi.org/10.3390/nu9030229

[7] Friso, S. and Choi, S.W. (2002) Gene-Nutrient Interactions and DNA Methylation. The Journal of Nutrition, 132, 2382S-2387S. https://doi.org/10.1093/jn/132.8.2382S

[8] Jariwalla, R.J., Niedwiecki, A. and Rath, M. (2010) Chapter 22-The Essentiality of Nutritional Supplementation in HIV Infection and AIDS: Review of Clinical Studies and Results from a Community Health Micronutrient Program. In: Bioactive Foods in Promoting Health: Fruits and Vegetables, Academic Press, Cambridge, 323-342. https://doi.org/10.1016/B978-0-12-374628-3.00022-0

[9] Strasser, F. and Bruera, E.D. (2002) Update on Anorexia and Cachexia. Hematologyl Oncology Clinics of North America, 16, 589-617. https://doi.org/10.1016/S0889-8588(02)00011-4

[10] Gallagher, P.G. (2017) Disorders of Erythrocyte Hydration. Blood, 130, 2699-2708. https://doi.org/10.1182/blood-2017-04-590810

[11] Richardson, W.S., Plaisance, A.M., Periou, L., Buquoi, J. and Tillery, D. (2009) Long-term Management of Patients after Weight Loss Surgery. Ochsner Journal, 9, 154-159.

[12] Al-Jafar, H., Al-Zamil, K., Al Ageeli, M., Alhaifi, M. and Al-Sabah, S. (2018) Potential Hematology and Nutritional Complications of Bariatric Surgery. Annals of Hematology \& Oncology, 5, 1209. https://doi.org/10.26420/annhematoloncol.2018.1209

[13] Brolin, R.E., LaMarca, L.B., Kenler, H.A. and Cody, R.P. (2002) J Malabsorptive Gastric Bypass in Patients with Superobesity. Journal of Gastrointestinal Surgery, 6, 195-203; discussion 204-205. https://doi.org/10.1016/S1091-255X(01)00022-1

[14] Bakshi, N. and Morris, C.R. (2016) The Role of the Arginine Metabolome in Pain: Implications for Sickle Cell Disease. Journal of Pain Research, 9, 167-175. https://doi.org/10.2147/JPR.S55571 
[15] Prakash, A. and Borgstahl, G.E. (2012) The Structure and Function of Replication Protein A in DNA Replication. Subcellular Biochemistry, 62, 171-196. https://doi.org/10.1007/978-94-007-4572-8_10

[16] Adjepong, K.O., Otegbeye, F. and Adjepong, Y.A. (2018) Perioperative Management of Sickle Cell Disease. Mediterranean Journal of Hematology and Infectious Diseases, 10, e2018032. https://doi.org/10.4084/mjhid.2018.032 